\title{
Ultrasonic Inspection To Quantify Failure Pathologies Of Crimped Electrical Connections
}

\author{
K. Elliott Cramer ${ }^{1, a)}$, Daniel F. Perey ${ }^{1}$ and William T. Yost $^{1}$ \\ ${ }^{1}$ NASA Langley Research Center, MS231, Hampton, VA 23681 \\ ${ }^{\text {a)} C o r r e s p o n d i n g ~ a u t h o r: ~ k . e l l i o t t . c r a m e r @ n a s a . g o v ~}$
}

\begin{abstract}
Previous work has shown that ultrasonic inspection provides a means of assessing electrical crimp quality that ensures the electrical and mechanical integrity of an initial crimp before the installation process is completed. The amplitude change of a compressional ultrasonic wave propagating at right angles to the wire axis and through the junction of a crimp termination was shown to correlate with the results of destructive pull tests, which is a standard for assessing crimp wire junction quality. Of additional concern are crimps made at high speed assembly lines for wiring harnesses, which are used for critical applications, such as in aircraft. During high-speed assembly it is possible that many faulty crimps go undetected until long after assembly, and fail in service. The position and speed of the crimping jaw become factors as the high-speed crimp is formed. The work presented in this paper is designed to cover the more difficult and more subtle area of high-speed crimps by taking into account the rate change of the measurements. Building on the previous work, we present an analysis methodology, based on transmitted ultrasonic energy and timing of the first received pulse that is shown to correlate to the gauge of the crimp/ferrule combination and the position of the crimping jaw. Results demonstrating the detectability of a number of the crimp failure pathologies, such as missing strands, partially inserted wires and incomplete crimp compression, are presented. The ability of this technique to estimate crimp height, a mechanical measure of crimp quality, is discussed.
\end{abstract}

\section{INTRODUCTION}

A wire-crimp terminator is a fixture which consists of a mating end and a crimp ferrule, and is installed on the end of a wire. This establishes a secure mechanical and electrical connection between the wire and the terminator. A terminator is installed onto the end of a wire by removing a portion of the insulation to expose a prescribed length of stranded wire, and placing it inside the ferrule. The ferrule is deformed by a tool designed to force the wire surfaces and the ferrule's inner surface into intimate contact. This connection maintains a residual force by the ferrule on the wire to assure good mechanical and electrical continuity between the wire's end and the terminator.

Previous work focused on manual crimping tools and has shown that ultrasonic inspection provides a means of assessing crimp quality that ensures the electrical and mechanical integrity of an initial crimp before the installation process is completed. The amplitude change of a compressional ultrasonic wave propagating at right angles to the wire axis and through the junction of a crimp termination was shown to correlate with the results of a destructive pull test, which is a standard for assessing crimp wire junction quality. A quantitative measure of the quality of the crimped connection based on the ultrasonic energy transmitted (crimp attenuation factor), was defined and shown to respond accurately to crimp quality ${ }^{1,2}$. 
In the previous work all measurements were taken at the point of full compression of the crimp. The work presented in this paper covers the more difficult and more subtle area of automated crimping by taking into account the rate change of the measurements during the crimp compression cycle. Building on the previous work, we present an analysis methodology, based on transmitted ultrasonic energy and timing of the first received pulse that is shown to correlate to the gauge of the crimp/ferrule combination and the position of the crimping jaw. Results are presented demonstrating the detectability of a number of the crimp failure pathologies, such as missing strands, partially inserted wires and incomplete crimp compression. The ability of this technique to estimate crimp height, a mechanical measure of crimp quality, is also discussed.

\section{ULTRASONIC INSPECTION TECHNIQUE}

The technique presented here uses ultrasonic wave transmission through the ferrule-wire joint (perpendicular to the geometric axis) to inspect a mechanically crimped connection. Figure 1 shows a schematic of the basic components. The send and receive transducers are damped PZT-5 ultrasonic compressional wave transducers with $7.5 \mathrm{MHz}$ center frequency and $6.35 \mathrm{~mm}$ diameter (Panametrics Model A121S). Each transducer is aligned with the center crimping location of the crimp tool. Upon application of pressure, the jaw deforms the crimp ferrule around the wire with sufficient force to press the wire and ferrule surfaces into intimate mechanical contact. This allows transmission of the ultrasonic wave through the ferrule, through the electrical wire, and into the opposite jaw to be received by an ultrasonic transducer. As the pressure increases, conformation between the crimp tool jaws, the ferrule, and the enclosed wire strands improves, and more of the ultrasonic wave energy propagates through the ferrule-wire joint.

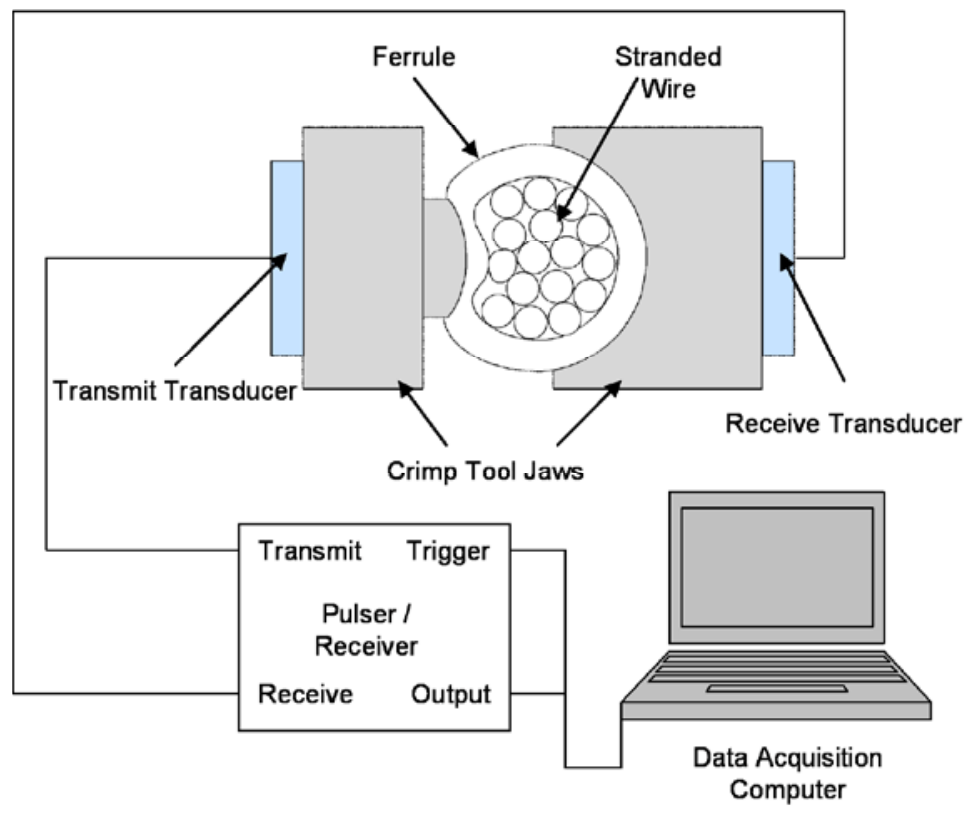

Figure 1. Schematic of the arrangement for ultrasonic interrogation of crimp connector.

The experimental setup consists of a commercial ultrasonic pulser-receiver (JSR Ultrasonics model PRC-50) in a pitch-catch arrangement as shown in Fig. 1. Data acquisition of the received ultrasonic time record was performed using a commercial 12-bit digitizer (Agilent-Acqiris model DP308) controlled by a laptop computer. The crimp tool is a modified, commercially available unit. The modifications to the crimp tool consisted of: (1) removing mounting holes for the crimp guide, (2) machining parallel external faces, (3) cutting small EDM notches and (4) machining the contact point at the jaw tip to minimize contact area. Modifications (3) and (4) were to eliminate (or reduce) any potential path for the ultrasound to propagate near the jaw tip. Figure 2 shows photographs of the commercially available crimp tool (Raychem Model 80-1377) and the tool modified by the manufacturer for these experiments. 


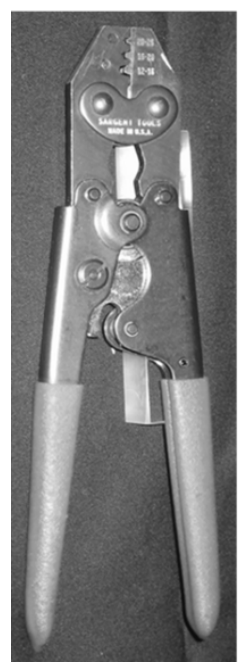

(a)

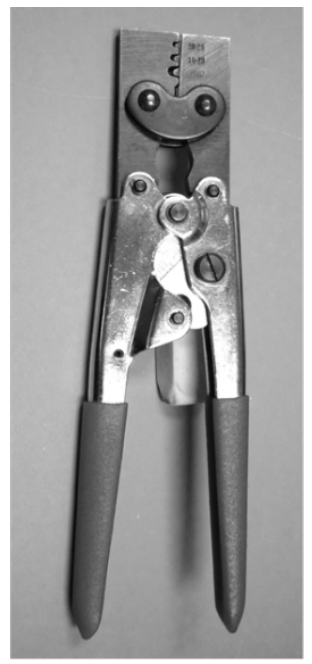

(b)

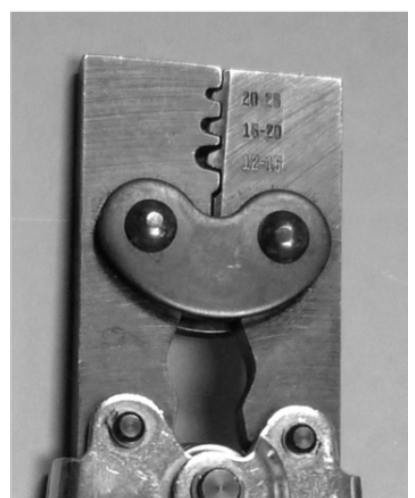

(c)

Figure 2. Photographs of (a) the commercially available crimp tool (Raychem Model 80-1377), (b) the tool modified for these experiments and (c) a close-up of the crimp jaws showing the details of the modifications (EDM notches not shown).

In order to simulate the action of an automatic crimping tool, the hand operated, instrumented crimp tool was mounted in a fixture that would allow the formation of crimps at controlled, repeatable increments of tool compression. Figure 3 shows a photograph of the fixture for controlling the crimp compression with the Panametrics Model A121S transducers affixed.

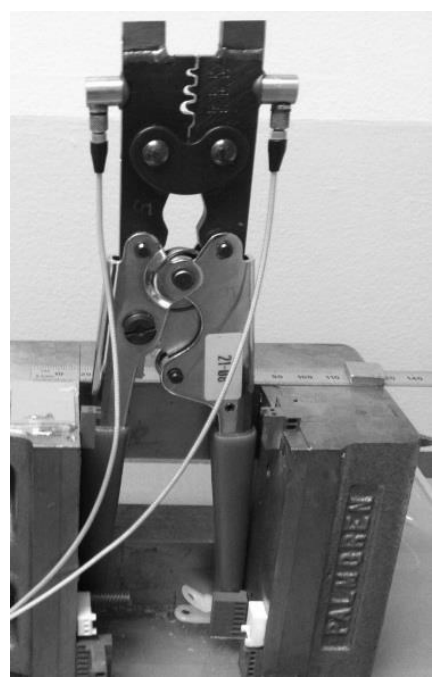

Figure 3. Photograph of the fixture for controlling the crimp compression with the Panametrics Model A121S transducers affixed

The wires used in these experiments were 16 AWG (American Wire Gauge) nickel plated copper (M22759/34). The insulation is stripped and inserted into a nickel plated, copper ferrules (MIL M81824/1) corresponding to the wire gauge and the crimping location on the tool. The ferrules are color coated such that red-banded ferrules accommodate 20-26 AWG wires, blue-banded ferrules accommodate 16-20 AWG wires and yellow-banded ferrules accommodate 12-16 AWG wires. For these experiments only the blue-banded ferrules were used. The blue-banded ferrule has a $2.69 \mathrm{~mm}$ minimum outside diameter and $1.62 \mathrm{~mm}$ minimum inside diameter. These terminators are used when making butt-splice type connections. The wire is inserted into the ferrule end of the terminator, and then the loaded ferrule is placed in the slot of the jaws of the instrumented crimp tool. The ultrasonic pulser-receiver is connected to the appropriate transducers, and the crimp tool is compressed while the ultrasonic sensors are active. 


\section{DATA COLLECTION AND ANALYSIS}

Using the experimental setup described previously, crimps are formed by compressing the handle of the crimp tool in $0.26 \mathrm{~mm}$ increments until full compression is achieved. At each increment, the output of the ultrasonic sensor is recorded by the digitizer as a separate time record. The data analysis consists of performing a Hilbert transform ${ }^{3}$ on each stored time record. Figure 4 (solid line) shows an example of the moduli of the Hilbert transformed data from one complete compression cycle, where the horizontal axis is the amount of handle compression in millimeters and the vertical axis is the amplitude of the ultrasonic signal in volts. Next, the moduli of the Hilbert transforms are integrated (summed) over the time window for each stored time record. The time window was selected to correspond to the full-width of the Hilbert transform at one half its maximum value for the main peak (first received ultrasonic pulse). This yields a value proportional to the ultrasonic energy transmitted through the crimp ${ }^{4}$. Figure 4 (dashed line) show the integrated (summed) values of the Hilbert transforms as a function of the amount of handle compression in millimeters.

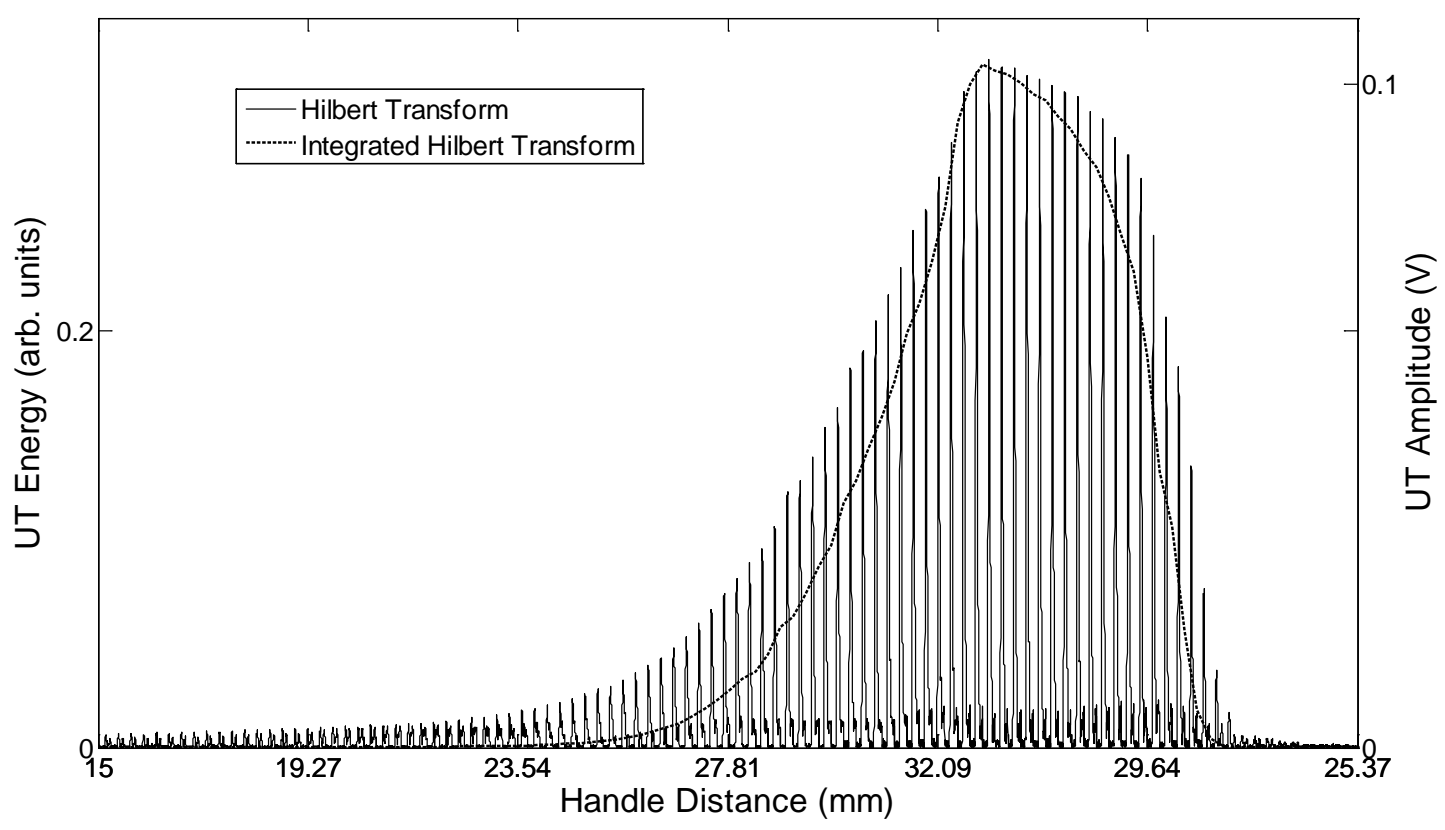

Figure 4. Moduli of the Hilbert transforms (solid line) of one complete compression cycle and time window integration of the moduli (dashed line).

The wires used in these experiments have 19 strands of 29 AWG (0.286 mm dia.) wire. Data are next acquired on crimps with a particular crimp junction defect pathology, specifically one and three strands (out of 19) removed. Figure 5 shows the integrated (summed) values of Hilbert transforms as a function of the amount of handle compression in millimeters for a good crimp and the defect cases. Note that for this case the horizontal axis uses full handle compression as the zero reference point with negative values indicating handle compression and positive values handle release after full compression. As expected from previous work ${ }^{2}$, the ultrasonic energy decreases in the presence of missing strands.

Finally, a series of 16 AWG crimps are formed, but the crimping process is stopped prior to full compression. Crimps are formed with crimp tool handle compressions of 5, 10, 15, 20 and 25mm less than full compression. Using a Schleuniger, Inc. Micrograph System, each of the crimps is sectioned through the region of largest compression, polished, electolytically stained and photographed. Figure 6 contains three representative micrographs showing two under compressed crimps (a) and (b) and one fully compressed crimp (c). From the micrographs, a measurement of the crimp height (in $\mathrm{mm}$ ), from the crown of crimp perpendicular to a line connecting the two lowest points, is made ${ }^{5}$. Table 1 shows the crimp height measurements and normalized ultrasonic energy for a fully compressed crimp and the five under compressed crimps. 


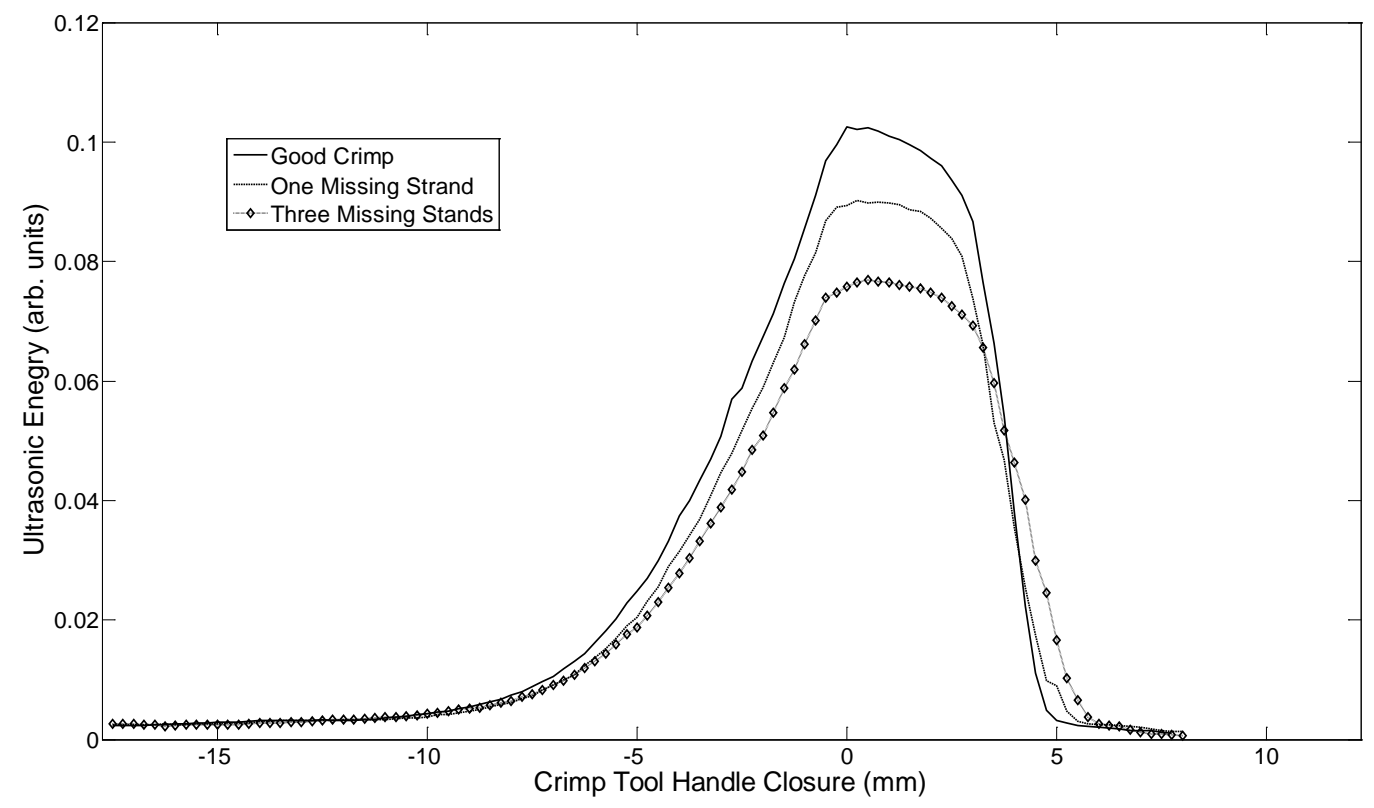

Figure 5. Integrated Hilbert transform vs. crimp tool handle closure for a good crimp and two defect pathologies (one and three missing strands). Note that full handle (crimp) compression is zero on the horizontal axis.

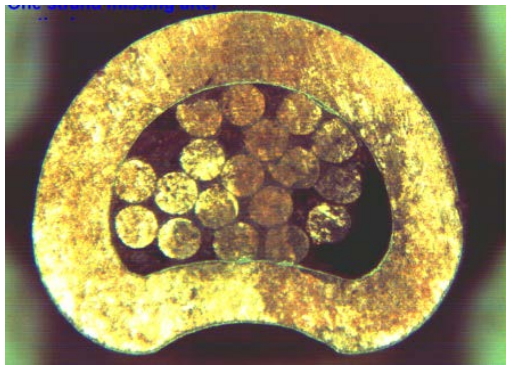

(a)

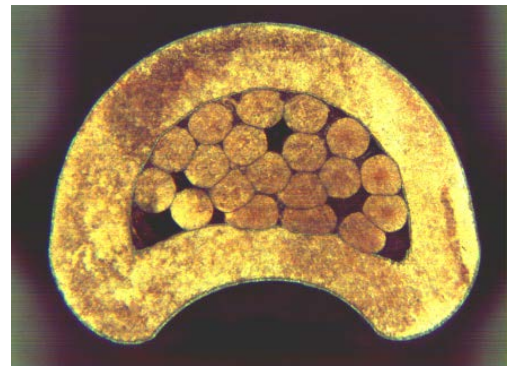

(b)

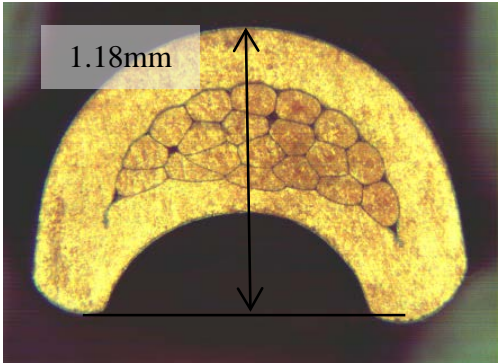

(c)

Figure 6. Micrographs showing representative crimps stopped short of full compression used for determining crimp heights. (a) $20 \mathrm{~mm}$ handle closure short of full compression (Note: One strand fell out during sectioning), (b) 10mm handle closure short of full compression and (c) full compression.

Table 1. Comparison of handle compression, crimp height (measured from micrographs) and normalized ultrasonic energy for a fully compressed and 5 under compressed crimps.

\begin{tabular}{ccc}
\hline Handle Compression & Crimp Height (mm) & $\begin{array}{c}\text { Normalized ultrasonic } \\
\text { energy }\end{array}$ \\
\hline Full & 1.18 & 1.000 \\
$-5 \mathrm{~mm}$ & 1.25 & 0.828 \\
$-10 \mathrm{~mm}$ & 1.30 & 0.655 \\
$-15 \mathrm{~mm}$ & 1.35 & 0.495 \\
$-20 \mathrm{~mm}$ & 1.40 & 0.371 \\
$-25 \mathrm{~mm}$ & 1.45 & 0.246 \\
\hline
\end{tabular}




\section{CONCLUSIONS}

We report here a new measurement technique to assess quantitatively the quality of wire crimp terminations during terminator installation. An apparatus has been developed to simulate automated crimping, and was used to acquire data to examine good crimps, under compressed crimps and those with missing wire strands (up to 3 strands out of 19). The modulus of the Hilbert Transform of the received transmission ultrasonic waveform data with integration over a time window, corresponding to the full-width at half maximum amplitude, as a function of crimp tool handle compression, has been shown in this paper to produce a reproducible, quantitative measure of crimp quality for the cases examined.

\section{ACKNOWLEDGMENTS}

The authors would like to thank John Callahan of Northrop Grumman Corporation for his help in laboratory setup and data acquisition.

\section{REFERENCES}

1. K. E. Cramer, D.F. Perey and W.T. Yost. NASA TP-2008-215348, 2008.

2. K.E. Cramer, D.F. Perey and W.T. Yost, "A Method For The Verification Of Wire Crimp Compression Using Ultrasonic Inspection,” Research in Nondestructive Evaluation, 2010, 21, 1, 18-29.

3. J. W. Goodman. Statistical Optics. John Wiley \& Sons, USA, 1985.

4. P. M. Gammell, "Improved ultrasonic detection using the analytic signal magnitude," Ultrasonics, vol 19, 1981, p73.

5. J. Camillo, “Assessing Crimp Quality,” in Assembly Magazine, 2012, http://www.assemblymag.com/articles/90259-assessing-crimp-quality (accessed July 7, 2014). 